\title{
IMMUNITY BOOSTING MEDICINAL PLANTS TO BEAT COVID -19 IN SERAJ BLOCK OF MANDI DISTRICT, HIMACHAL PRADESH
}

\author{
Manju Lata ${ }^{1}$ (iD) Mohammad Jamali ${ }^{2}$ \\ ${ }^{1}$ Department of Biosciences MLSM College Sunder Nagar, District Mandi, Himachal Pradesh, India. \\ ${ }^{2}$ Deputy Head, Khawarizmi International College, Abu Dhabi, UAE.
}

\section{ARTICLE INFO}

Received: 29 August 2021

Revised:06 November 2021

Accepted: 08 November 2021

Keywords:

COVID-19, Immunity Boosting, Medicinal Plants, Seraj Block.

Corresponding Author:

Manju Lata

Email:

manjulata18@gmail.com

Copyright () 2021 by author(s) and Journal of Scientific Research in Medical and Biological Sciences.

This work is licensed under the Creative Commons Attribution International License (CC BY 4.0). http://creativecommons.org/licenses/ by/4.0/

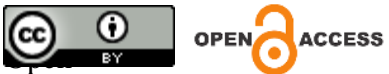

\begin{abstract}
Purpose: This study aimed to document the present status of medicinal plants used to boost the immunity to combat Coronavirus.

Materials and Methods: For the assessment and use of herbs during the COVID 19 pandemic, surveys were conducted in different villages of Seraj block. The surveys were conducted during the spread of the pandemic from 2020 to 2021. Local people were interviewed and information on the utilization of local herbs during this pandemic was recorded. Information on parts used, the procedure of use, habit, habitat, etc. was also collected.

Results: A total of 58 species of medicinal plants from 27 families and 42 genera were documented. Among them, the most dominant family was Rosaceae. Most of the plants such as Morchella sp, Naustratium officinale,Taraxacum officinale, Urtica dioica ,Allium sp, Withania somnifera, Curcuma longa, Cannabis, Mentha, Ocimum sanctum are rich in antioxidants and have a wide range of medicinal values used to treat cough, cold, fever, and bronchitis. All these plants were used traditionally to boost immunity. Due to the covid -19 pandemic, the utilization of these herbal plants has increased many folds in the area. These medicinal plants should be made available to scientists to design clinical trials. Integration of this concept would certainly develop drug therapy in the near future.

Conclusion: The study found that the use of medicinal plants has increased during COVID-19 and most of the respondents recommended medicinal plants to prevent COVID-19 and to boost immunity. More studies should be conducted to develop certain formulations.
\end{abstract}

\section{INTRODUCTION}

The Indian Himalayan Region (IHR) covers approximately 4,19,873 km2 area (Rodgers \& Panwar, 1988), and covers 10 states namely, Jammu \& Kashmir, Himachal Pradesh, Uttarakhand, Sikkim, Arunachal Pradesh, Meghalaya, Nagaland, Manipur, Mizoram, Tripura, and hill regions of 2 states viz. Assam and West Bengal of Indian Republic. The study was carried out in the Seraj block of District Mandi Himachal Pradesh. Due to diverse habitats and large altitudinal range (2000-3500), it supports unique and socio-economically important floristic diversity. It has also been playing an important role in increasing the economic level of people as Seraj exports medicinal plants to different parts of the country and world. The elder people living in rural areas have more knowledge of traditional medicine. The new coronavirus disease (COVID-19) pandemic has caused global socioeconomic disturbances with a worrisome number of deaths and health issues, and the world has been struggling to find medicine to treat 
and prevent COVID-19 (Raghuvir, 2000). A number of combinations and trials have been done, but so far, they have not produced promising results(Boulware et al., 2000). There is a strong inter-relationship between people and plants according to needs. People are dependent on plants for different purposes such as for food, medicine, and houses. Plant species have always been a fundamental source for the discovery of drugs. People had used medicinal plants to fight against pandemics in the past (Arora et al., 2011), and dependency of people on medicinal plants might have increased these days around the world as medicinal plants can be an alternative option to prevent COVID-19. Therefore, this study presents an examination of some medicinal plants that can be used to combat coronavirus. Different researchers have suggested herbal medicine as a potential option to cure or prevent COVID-19 (Vellingiri et al., 2020). Countries like China and India are integrating their use with western medicine to boost the immunity power of COVID-19 patients. On the other hand, the World Health Organization (WHO) (2020) claims medicinal plants might be good for the health and in supporting the immune system, but not in preventing or curing COVID-19.

\section{METHODOLOGY}

\section{Study area}

Himachal Pradesh $\left(30^{\circ} 22^{\prime} 40^{\prime \prime}\right.$ to $30^{\circ} 12^{\prime} 40^{\prime \prime} \mathrm{N}$ latitudes and $75^{\circ} 47^{\prime} 55^{\prime \prime}$ to $79^{0} 04^{\prime} 20^{\prime \prime} \mathrm{E}$ longitudes) is a Northwestern Himalayan state of India which is a rich repository of ethnomedicinal flora. Most of these plant species find their use in traditional medicine, folk uses, and also in modern industry (Singh \& Thakur, 2014; Kumar\& Jamwal, 2021). The present study was carried out in Seraj block of district Mandi $\left(31.5892^{\circ} \mathrm{N}, 76.9182^{\circ} \mathrm{E}\right)$ Himachal Pradesh. The total population of the Seraj block is 1,06,444 with 469 Villages and 59 Panchayats (Statistical Department Mandi). The area is covered by a dense forest of conifers and oak trees. This area is rich in medicinal flora. Soil is fertile and rich in humus and nitrogenous compounds but lacks phosphate compounds. The major soil groups are brown hill soil and red loamy soil. Most soil in this region is acidic in nature. Being a hilly valley climate is cool and temperate with three distinct seasons; winter (October to March ), summer (April to June), monsoon (July to September). The highest temperature is recorded during May and June varying between 30 to 35 . The lowest temperature is recorded during December and January month. The annual rainfall is around 1240mm. (Lata \& Kumar, 2020; Lata, 2020).

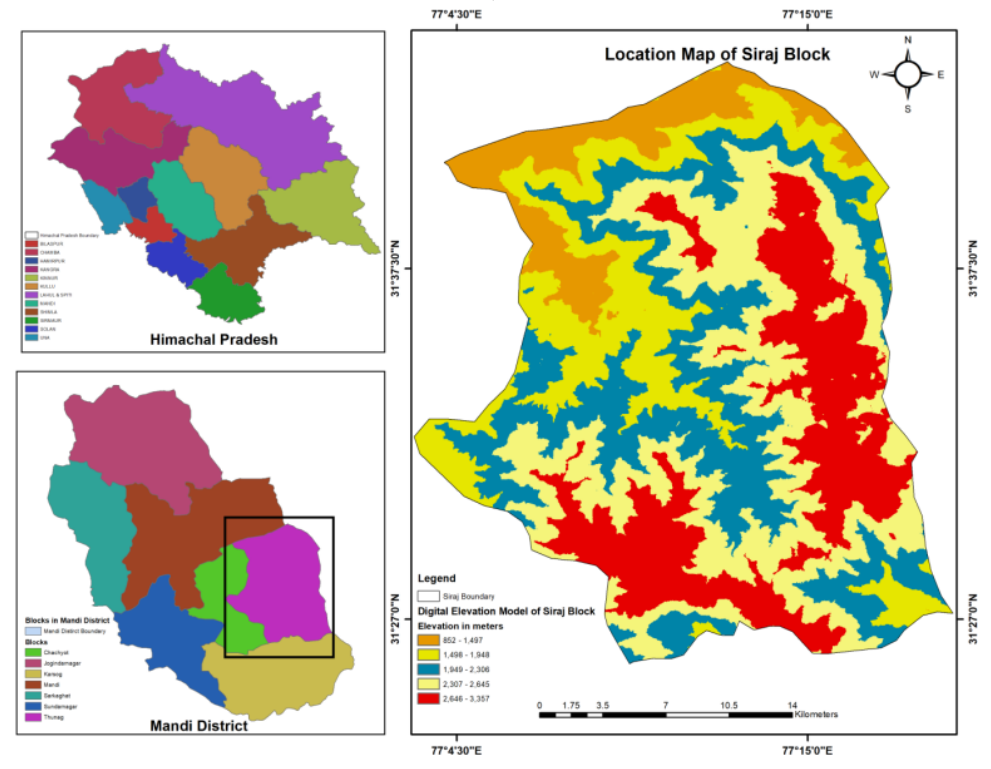

\section{5 |}




\section{Methodology}

For the assessment and use of herbs during the COVID 19 pandemic, surveys were conducted in different villages of Seraj block, namely Shilibagi, Cheung, Didier, Suragi, Chendi, Keuli, Lambatach, Bakhlwar, Jarol, Kothi, Rod, Chapper, Majakhal, Kutah, Dhanshali, Tungadhar, Bhanvas, Danhyar, Kataru, Sanglwara, Dusadhi, Dhrut, Kheladhar, Ropa, Surah, Murag. Surveys were conducted during the spread of the pandemic from 2020 to 2021. Local people were interviewed and information on the utilization of local herbs during this pandemic was recorded. The interviews were mostly individual. Interviews followed the informal method and open-ended rather than a strict questionnaire. The language used while interacting with the informants was the local dialect of the study area viz. Pahari and in certain cases, Hindi also. Information on parts used, the procedure of use, habit, habitat, etc. was also collected.

Table 1: Demographic profile of informants: $\mathrm{N}=150$

\begin{tabular}{llll}
\hline Parameter & Description & Total Respondents & Frequency \% \\
\hline & $21-30$ & 12 & $8 \%$ \\
& $31-40$ & 32 & $21.3 \%$ \\
Age & $41-50$ & 27 & $18 \%$ \\
& $51-60$ & 29 & $19.3 \%$ \\
& $61-70$ & 34 & $22.7 \%$ \\
Sex & $71-80$ & 16 & $10.7 \%$ \\
& Female & 80 & $53.4 \%$ \\
& Male & 70 & $46.6 \%$ \\
\hline
\end{tabular}

RESULTS AND DISCUSSION

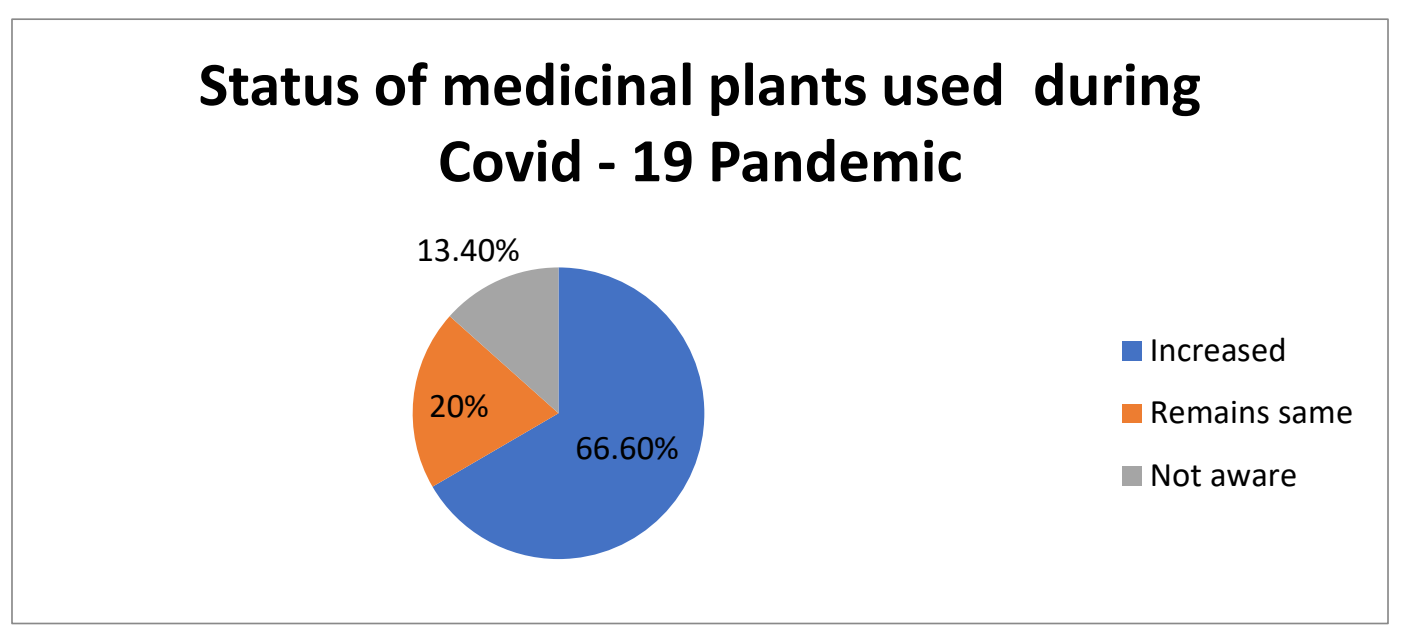

Fig :1 Status of medicinal plants used

Out of 150 respondents, $100(66.6 \%)$ respondents agreed that the use of the medicinal plant has increased during COVID-19, whereas $30(20 \%)$ agreed the use of medicinal plants during COVID-19 is the same as that of normal condition, 20 (13.4\%) respondents were not aware. 
Table 2: Immunity-boosting medicinal plants to beat COVID -19 in Seraj Block of District Mandi.

\begin{tabular}{|c|c|c|c|c|c|c|}
\hline Family/Taxa & Local Name & $\begin{array}{l}\text { Altitudinal } \\
\text { Range (m.) }\end{array}$ & $\begin{array}{c}\text { Life } \\
\text { Form } \\
\end{array}$ & $\begin{array}{l}\text { Part } \\
\text { Used }\end{array}$ & Nativity & Indigenous Use by inhabitants \\
\hline \multicolumn{7}{|l|}{ Angiosperm } \\
\hline \multicolumn{7}{|l|}{ Amaranthaceae } \\
\hline Achyranthes aspera L. & $\begin{array}{l}\text { Putkanda/ } \\
\text { Umalkudi }\end{array}$ & $1800-2500$ & $\mathrm{H}$ & $\mathrm{Lf}$ & As Trop & Leaves cooked to make sag. \\
\hline \multicolumn{7}{|l|}{ Anacardiaceae } \\
\hline $\begin{array}{l}\text { Pistacia chinensis } \\
\text { subsp. integerrima (J. } \\
\text { L. Stewart ex Brandis) } \\
\text { Rech. f. }\end{array}$ & $\begin{array}{l}\text { Kakare/ Kakar } \\
\text { singhi }\end{array}$ & $1800-2200$ & $\mathrm{~T}$ & $\mathrm{Fr}$ & $\begin{array}{l}\text { Reg Himal } \\
\text { Egypt Persia }\end{array}$ & $\begin{array}{l}\text { Fruits are burn in the fire and } \\
\text { powder are consumed during } \\
\text { persistent cough. }\end{array}$ \\
\hline Rhus javanica L.** & Titri & $1800-2200$ & $\mathrm{~T}$ & $\mathrm{Fr}$ & Reg Himal & Fruits are eaten. \\
\hline \multicolumn{7}{|l|}{ Apiaceae } \\
\hline $\begin{array}{l}\text { Chaerophyllum } \\
\text { reflexum Lindl. }\end{array}$ & Khelti & $2100-3369$ & $\mathrm{H}$ & Rt & Reg Himal & $\begin{array}{l}\text { Roots are used for salad and } \\
\text { vegetables. }\end{array}$ \\
\hline $\begin{array}{l}\text { C.villosum Wall. ex } \\
\text { DC.** }\end{array}$ & Jangli gazar & $2500-3369$ & $\mathrm{H}$ & WP & Reg Himal & $\begin{array}{l}\text { Roots are used for salad and } \\
\text { vegetables. }\end{array}$ \\
\hline $\begin{array}{l}\text { Heracleum candicans } \\
\text { L. }\end{array}$ & Badiyacha & $1800-2800$ & $\mathrm{H}$ & Lf, Rt & Reg Himal & Stem and root are eaten. \\
\hline \multicolumn{7}{|l|}{ Alliaceae } \\
\hline Allium humile & Lahne & 2000-3000 & $\mathbf{H}$ & $\begin{array}{l}\text { Bl, } \\
\text { Lf. }\end{array}$ & & $\begin{array}{l}\text { Raw soaked in water,Dried, boil } \\
\text { with water,leaves cooked, eaten as } \\
\text { salad }\end{array}$ \\
\hline $\begin{array}{l}\text { Amaryllidaceae } \\
\text { Allium sativum L. }\end{array}$ & Lasun & $1500-3000$ & $\mathrm{H}$ & $\begin{array}{l}\text { Bl } \\
\text { Lf }\end{array}$ & & $\begin{array}{l}\text { Raw soaked in water,Dried, boil } \\
\text { with water }\end{array}$ \\
\hline $\begin{array}{l}\text { Myriactis nepalensis } \\
\text { Less. }\end{array}$ & - & $1800-3369$ & $\mathrm{H}$ & $\mathrm{Lf}$ & $\begin{array}{l}\text { Reg Himal } \\
\text { As Centr }\end{array}$ & $\begin{array}{l}\text { Leaves and young shoots are } \\
\text { cooked. Roasted fruits are pickled } \\
\text { also. }\end{array}$ \\
\hline $\begin{array}{l}\text { Taraxacum officinalis } \\
\text { Webb. }\end{array}$ & Gahri phool & $1800-2800$ & $\mathrm{H}$ & WP & Am Trop & $\begin{array}{l}\text { Leaves cooked as vegetables. } \\
\text { Leaves are eaten as a raw in soup } \\
\text { or salad }\end{array}$ \\
\hline \multicolumn{7}{|l|}{ Balsaminaceae } \\
\hline $\begin{array}{l}\text { Impatiens glandulifera } \\
\text { Royle }\end{array}$ & - & $2200-3369$ & $\mathrm{H}$ & $\mathrm{Sd}, \mathrm{Fl}$ & Reg Himal & $\begin{array}{l}\text { Seeds, young leaves, shoots are } \\
\text { cooked and eaten. Flowers are } \\
\text { turned into a jam. }\end{array}$ \\
\hline I. racemosa Hk. f.** & - & $1800-2700$ & $\mathrm{H}$ & $\mathrm{Sd}, \mathrm{AP}$ & Reg Himal & $\begin{array}{l}\text { Leaves and young shoots are } \\
\text { cooked. Seeds are consumed raw } \\
\text { or cooked. }\end{array}$ \\
\hline I. scabrida DC.** & - & $1800-2600$ & $\mathrm{H}$ & $\mathrm{Sd}, \mathrm{WP}$ & Reg Himal & $\begin{array}{l}\text { Aerial parts cooked and consumed } \\
\text { as vegetables. }\end{array}$ \\
\hline I. sulcata Wall.** & - & $2000-3369$ & $\mathrm{H}$ & $\mathrm{Sd}, \mathrm{AP}$ & Reg Himal & $\begin{array}{l}\text { Seeds are pickled and aerial parts } \\
\text { cooked. }\end{array}$ \\
\hline \multicolumn{7}{|l|}{ Berberidaceae } \\
\hline $\begin{array}{l}\text { Berberis aristata } \\
\text { DC.** }\end{array}$ & Kasmal & $1800-2800$ & $\mathrm{Sh}$ & WP & Ind Or & Ripe fruits are eaten \\
\hline $\begin{array}{l}\text { B.jaeschkeana } \\
\text { Sehneid.** }\end{array}$ & Kasmal & $2700-3369$ & $\mathrm{Sh}$ & $\mathrm{Rt}, \mathrm{Fr}$ & Reg Himal & Ripe fruits are eaten \\
\hline B. lycium Royle** & Kasmal & $1800-2200$ & $\mathrm{Sh}$ & Fr & Reg Himal & Ripe fruits are eaten \\
\hline \multicolumn{7}{|l|}{ Brassicaceae } \\
\hline $\begin{array}{l}\text { Nasturtium officinale } \\
\text { R.Br. }\end{array}$ & Chucch & $2000-3000$ & $\mathrm{H}$ & Lf & - & $\begin{array}{l}\text { Leaves and tender twigs are cooked } \\
\text { as vegetable or eaten as raw in the } \\
\text { form of salads. }\end{array}$ \\
\hline Cannabaceae & & & & & & \\
\hline
\end{tabular}




\begin{tabular}{|c|c|c|c|c|c|c|}
\hline Cannabis sativa $\mathrm{L}$. & Bijya & $1800-2000$ & $\mathrm{H}$ & $\begin{array}{l}\text { Lf, Sd, } \\
\text { Fr, Fl }\end{array}$ & $\begin{array}{lr}\text { As } & \text { Centr } \\
\text { Reg } & \text { Himal } \\
\text { Bor } & \text { Occ }\end{array}$ & $\begin{array}{l}\text { Its seeds are grinded and salt is } \\
\text { prepared .Seeds are used as a } \\
\text { stuffing material for Siddu and } \\
\text { Kauchari.Leaf in small quantity are } \\
\text { used to make pakora. }\end{array}$ \\
\hline \multicolumn{7}{|l|}{ Dioscoreaceae } \\
\hline $\begin{array}{l}\text { Dioscorea deltoidea } \\
\text { Wall. ex Kunth }\end{array}$ & - & $1800-2800$ & $\mathrm{H}$ & $\mathrm{Tu}$ & Ind & Tubers are cooked and eaten. \\
\hline \multicolumn{7}{|l|}{ Elaeagnaceae } \\
\hline $\begin{array}{l}\text { Elaeagnus parvifolia } \\
\text { Wall. ex Royle }\end{array}$ & Geai & $1800-2400$ & $\bar{T}$ & Fr & Japan & Fruits are edible. \\
\hline \multicolumn{7}{|l|}{ Ericaceae } \\
\hline $\begin{array}{l}\text { Rhododendron } \\
\text { arboretum }\end{array}$ & Burah & $2000-3000$ & $\mathrm{~T}$ & fl. & $\begin{array}{l}\text { Ind Or Reg } \\
\text { Himal } \\
\text { Zeylan }\end{array}$ & $\begin{array}{l}\text { Flowers are grinded and chutney } \\
\text { prepared from them and consumed } \\
\text { during summer season. Fresh juice } \\
\text { and sharbat are also made from the } \\
\text { flowers. }\end{array}$ \\
\hline $\begin{array}{l}\text { Fabaceae / Trigonella } \\
\text { foenum-graecum } \mathrm{L} .\end{array}$ & Methi & $1500-3000$ & $\mathrm{H}$ & Sd,lf & & $\begin{array}{l}\text { Decoction of Seed or leaves used } \\
\text {,seed also added while preparing } \\
\text { tea .Seeds are soaked in water and } \\
\text { left whole night ,soaked water } \\
\text { consumed. }\end{array}$ \\
\hline \multicolumn{7}{|l|}{ Grossulariaceae } \\
\hline Ribes glaciale Wall. & - & $2800-3369$ & $\mathrm{Sh}$ & $\mathrm{Fr}$ & Reg Himal & The ripe fruit is edible. \\
\hline \multicolumn{7}{|l|}{ Hippocastanaceae } \\
\hline $\begin{array}{l}\text { Aesculus indica Coleb. } \\
\text { ex Camb.** }\end{array}$ & Khanor & $1800-2600$ & $\mathrm{~T}$ & Fr & Reg Himal & $\begin{array}{l}\text { Fruits are soaked in water then } \\
\text { grinded and flour is obtained which } \\
\text { is known as seek .from the seek } \\
\text { rotis are made. }\end{array}$ \\
\hline \multicolumn{7}{|l|}{ Juglandaceae } \\
\hline Juglans regia $\mathrm{L} . * *$ & Akhrot & $1800-2000$ & $\mathrm{~T}$ & $\mathrm{Fr}$ & $\begin{array}{l}\text { As Occ Reg } \\
\text { Himal }\end{array}$ & $\begin{array}{l}\text { Fruits are used as a stuffing } \\
\text { material for siddu. After harvesting } \\
\text { fruits are dried and stored for future } \\
\text { use as a dry fruits. Green leaves and } \\
\text { tender twigs are used as a (datun) } \\
\text { to clean teeth }\end{array}$ \\
\hline \multicolumn{7}{|l|}{ Lamiaceae } \\
\hline Mentha arvensis $\mathrm{L}$. & Pudina & $1500-3000$ & $\mathrm{H}$ & Lf & Europe & $\begin{array}{l}\text { Powder, boil with water, paste } \\
\text { consumed. }\end{array}$ \\
\hline $\begin{array}{l}\text { Elsholtzia fruticosa } \\
\text { (Don) Rehd. }\end{array}$ & Bothi, & $1800-2200$ & $\mathrm{H}$ & $\mathrm{Lf}, \mathrm{Fr}, \mathrm{Sd}$ & Reg Himal & $\begin{array}{l}\text { The powdered seeds are used as a } \\
\text { condiment for flavouring } \\
\text { foodstuffs }\end{array}$ \\
\hline $\begin{array}{l}\text { Thymus linearis } \\
\text { Benth. }\end{array}$ & $\begin{array}{l}\text { Ban } \\
\text { jira/madroshda }\end{array}$ & $2000-3369$ & $\mathrm{H}$ & WP & $\begin{array}{l}\text { Europe As et } \\
\text { Afr Bor }\end{array}$ & $\begin{array}{l}\text { Aerial parts of plants are wrapped } \\
\text { with in a cotton cloth and used to } \\
\text { foment with hot water or heat, } \\
\text { which relieve the stomach ache of } \\
\text { infants as well as adults. }\end{array}$ \\
\hline Ocimum sanctum & Tulsi & $1500-2800$ & $\mathrm{H}$ & WP & $\begin{array}{l}\text { Ind sub } \\
\text { con }\end{array}$ & $\begin{array}{l}\text { Decoction of leaves ,seeds } \\
\text { consumed.Powdered seeds and } \\
\text { leaves used in beverages. }\end{array}$ \\
\hline \multicolumn{7}{|l|}{ Liliaceae } \\
\hline $\begin{array}{l}\text { Polygonatum } \\
\text { verticillatum (L.) Al. }\end{array}$ & Salam-Mishri & $2000-3369$ & $\mathrm{H}$ & $\mathrm{Tu}$ & $\begin{array}{l}\text { Europe } \\
\text { As Bor }\end{array}$ & $\begin{array}{l}\text { Its paste is applied on forehead } \\
\text { during high fever. }\end{array}$ \\
\hline $\begin{array}{l}\text { Myrtaceae } \\
\text { Syzygium cumini }\end{array}$ & Jamun & $2500-3000$ & $\mathrm{~T}$ &. $\mathrm{Fr}$ & Reg Himal & Ripe fruits eaten as a raw \\
\hline Ficus nemoralis & Dudla & $1800-2000$ & $\bar{T}$ & Fr, Lf & Reg Himal & Leaves are cooked as a vegetable. \\
\hline
\end{tabular}




\begin{tabular}{|c|c|c|c|c|c|c|}
\hline Morus nigra & Cheemu & $1800-2200$ & $\mathrm{~T}$ & Fr & Reg Himal & $\begin{array}{l}\text { Ripe fruits are very delicious and } \\
\text { consumed. }\end{array}$ \\
\hline \multicolumn{7}{|l|}{ Myricaceae } \\
\hline Myrica esculenta & Kafal & $1800-2500$ & $\mathrm{~T}$ & fr & Reg Himal & Ripe fruits are consumed. \\
\hline \multicolumn{7}{|l|}{ Oxalidaceae } \\
\hline $\begin{array}{l}\text { Oxalidaceae } \\
\text { Oxalis } \\
\text { corniculata }\end{array}$ & Malori & $2500-3000$ & $\mathrm{H}$ & Lf ,fl & Reg Himal & $\begin{array}{l}\text { Leaves consumed to make } \\
\text { chutney.Infusion consumed. }\end{array}$ \\
\hline \multicolumn{7}{|l|}{ Polygonaceae } \\
\hline $\begin{array}{l}\text { Fagopyrum debotrys } \\
\text { (Don) Hara }\end{array}$ & Fafra & $1800-2800$ & $\mathrm{H}$ & $\mathrm{Lf}$ & $\begin{array}{l}\text { Reg Himal } \\
\text { China }\end{array}$ & $\begin{array}{l}\text { Leaves are edible consumed as } \\
\text { green leafy vegetables. }\end{array}$ \\
\hline F. esculentum Moench & Oghal & $2000-3000$ & $\mathrm{H}$ & $\overline{\mathrm{Lf}}$ & $\begin{array}{l}\text { Europe As } \\
\text { Bor }\end{array}$ & $\begin{array}{l}\text { Leaves are cooked as vegetables. } \\
\text { Seeds are grinded into powder and } \\
\text { flour is made which in turn is used } \\
\text { to make bread or mixed with wheat } \\
\text { flour or others. }\end{array}$ \\
\hline $\begin{array}{l}\text { Persicaria nepalense } \\
\text { Meisn. }\end{array}$ & Nalora & $1800-2000$ & $\mathrm{H}$ & $\mathrm{Lf}$ & $\begin{array}{lr}\text { As et } & \text { Afr } \\
\text { Trop } & \text { et } \\
\text { Subtrop } & \\
\end{array}$ & $\begin{array}{l}\text { Leaves and tender shoots are } \\
\text { cooked. }\end{array}$ \\
\hline \multicolumn{7}{|l|}{ Rosaceae } \\
\hline $\begin{array}{l}\text { Cotoneaster } \\
\text { microphyllus Wall. ex } \\
\text { Lindl. }\end{array}$ & Chinchri & $2000-3369$ & Sh & Rt, Lf, Fr & Reg Himal & Ripe fruits are eaten. \\
\hline Fragaria nubicola $\mathrm{L}$. & Ghumpal & $2000-3000$ & $\mathrm{H}$ & Fr & Reg Temp & $\begin{array}{l}\text { Raw fruits are eaten. They are } \\
\text { very tasty with strawberry flavor. }\end{array}$ \\
\hline Prinsepia utilis Royle & Bekhal & $1800-2600$ & $\mathrm{Sh}$ & $\mathrm{Sd}, \mathrm{Fr}$ & Reg Himal & $\begin{array}{l}\text { An edible oil is obtained from the } \\
\text { seed used in cooking. }\end{array}$ \\
\hline $\begin{array}{l}\text { Prunus cerasoides } \\
\text { Don }\end{array}$ & Paja & $1800-2200$ & $\mathrm{~T}$ & Fr & Reg Himal & $\begin{array}{l}\text { Ripe fruits are edible and its leaves } \\
\text { have religious value used to } \\
\text { worship the deities. }\end{array}$ \\
\hline $\begin{array}{l}\text { Pyrus pashia } \text { Buch.- } \\
\text { Ham. ex Don }\end{array}$ & Kainth & $1800-2200$ & $\mathrm{~T}$ & Fr & Reg Himal & $\begin{array}{l}\text { Ripe fruits are very delicious and } \\
\text { are eaten by inhabitants. }\end{array}$ \\
\hline Rosa brunonii Lindl. & Kuja/ Shami & $1800-2200$ & $\mathrm{Sh}$ & Fr & $\begin{array}{l}\text { Reg Himal } \\
\text { China }\end{array}$ & Fruits are eaten. \\
\hline R. sericea Lindl. & - & $2000-3369$ & Sh & Rt, Fr, Fl & Reg Himal & Fruits and seeds are eaten. \\
\hline $\begin{array}{l}\text { Rubus biflorus Buch.- } \\
\text { Ham. ex Sm. }\end{array}$ & Akhaey & $1800-2500$ & $\mathrm{Sh}$ & Fr & Reg Himal & Ripe fruits are edible \\
\hline R. foliolosus D.Don & Akha & $2700-3369$ & $\mathrm{Sh}$ & Fr & Reg Himal & $\begin{array}{l}\text { Ripe fruits are edible, rich source of } \\
\text { vitamin C. }\end{array}$ \\
\hline R.niveus Thunb. & - & $1800-2600$ & Sh & $\mathrm{Fr}$ & Reg Himal & $\begin{array}{l}\text { Ripe fruits are edible, rich source of } \\
\text { vitamin C. }\end{array}$ \\
\hline R. paniculatus Sm.** & $\begin{array}{l}\begin{array}{l}\text { Kalanche, Kala } \\
\text { akha }\end{array} \\
\end{array}$ & $1800-2600$ & Sh & Fr & Reg Himal & Ripe fruits are edible \\
\hline \multicolumn{7}{|l|}{ Saxifragaceae } \\
\hline $\begin{array}{l}\text { Astilbe rivularis } \\
\text { Buch.-Ham. ex Don }\end{array}$ & - & $1800-2800$ & $\mathrm{H}$ & $\mathrm{Lf}$ & Reg Himal & $\begin{array}{l}\begin{array}{l}\text { Young leaves are edible and } \\
\text { cooked. }\end{array} \\
\end{array}$ \\
\hline \multicolumn{7}{|l|}{ Solanaceae } \\
\hline Withania sominifera & Ashwagandha & $1200-2500$ & $\mathrm{H}$ & Lf & Europe & $\begin{array}{l}\text { Decotation of Leaf,powdered,tea } \\
\text { prepared. }\end{array}$ \\
\hline $\begin{array}{l}\text { Taxaceae /Taxus } \\
\text { baccata }\end{array}$ & Rakhal & $2500-3000$ & $\mathbf{T}$ & If & Reg Himal & $\begin{array}{l}\text { Decoction of leaves used ,dried } \\
\text {,powdered ,tea prepared. }\end{array}$ \\
\hline \multicolumn{7}{|l|}{ Urticaceae } \\
\hline $\begin{array}{l}\text { Urtica dioica Jacq. ex } \\
\text { Wedd. }\end{array}$ & Kugshi & $1800-2300$ & $\mathrm{H}$ & $\mathrm{Lf}$ & $\begin{array}{ll}\text { Reg } & \text { Bor } \\
\text { Temp } & \end{array}$ & $\begin{array}{l}\text { Tender leaves are chopped, cooked } \\
\text { and consumed as a vegetable. } \\
\text { Leaves are used to make chutney. }\end{array}$ \\
\hline Vitaceae & & & & & & \\
\hline
\end{tabular}




\begin{tabular}{|l|l|l|l|l|l|l|}
\hline $\begin{array}{l}\text { Parthenocissus } \\
\text { semicordata (Royle) } \\
\text { Planch.** }\end{array}$ & Kramru & $1800-2600$ & Sh & Fr & Ind Or & Sweet Juicy fruits are consumed. \\
\hline $\begin{array}{l}\text { Zingiberaceae } \\
\text { Curcuma longa }\end{array}$ & Haldi & $1500-3000$ & $\mathrm{H}$ & $\mathrm{Rz}$ & & $\begin{array}{l}\text { Boil with water or milk, raw, } \\
\text { powder taken with water or milk }\end{array}$ \\
\hline Fungi & & & & & & \\
\hline Morchellaceae & & & & & - & $\begin{array}{l}\text { Fruiting bodies are cooked and } \\
\text { eaten. }\end{array}$ \\
\hline $\begin{array}{l}\text { Morchella comica } \\
\text { Pers. }\end{array}$ & Guchhi/Dunglu & $1800-3000$ & & Frb & $\begin{array}{l}\text { Fruiting bodies are dried and sold } \\
\text { in the market due to its high } \\
\text { commercial value. It is also } \\
\text { consumed as a vegetable. }\end{array}$ \\
\hline $\begin{array}{l}\text { M. } \text { esculenta } \text { (L.) Pers. } \\
\text { Guchhi/Dunglu }\end{array}$ & $1800-3000$ & & Frb & & $\begin{array}{l}\text { Fruiting bodies are cooked and } \\
\text { eaten as a vegetable. It is known } \\
\text { for its delicacy. }\end{array}$ \\
\hline $\begin{array}{l}\text { Gyromitra esculenta } \\
\text { (Pers.) Fr. }\end{array}$ & $\begin{array}{l}\text { Ban } \\
\text { Dunglu/Guchhi }\end{array}$ & $2500-3300$ & & Frb & & - \\
\hline
\end{tabular}

Abbreviations used: $\mathrm{AR}=\mathrm{Altitudinal}$ range; $\mathrm{H}=\mathrm{Herb} ; \mathrm{Sh}=\mathrm{Shrub} ; \mathrm{T}=\mathrm{Tree} ; \mathrm{AP}=$ Aerial part; $\mathrm{Bk}=\mathrm{Bark} ; \mathrm{Fl}=\mathrm{Flower}$; $\mathrm{Fr}=$ Fruit; Infl= Inflorescence; $\mathrm{Lf}=\mathrm{Leaf} ; \mathrm{Rh}=\mathrm{Rhizome} ; \mathrm{Rt}=\mathrm{Root} ; \mathrm{Sd}=\mathrm{Seed} ; \mathrm{St}=\mathrm{Stem} ; \mathrm{Tu}=$ Tuber; WP= Whole plant; Wd= Wood; Res= Resin; Frd= Frond; Frb= Fruiting body; La=Latex; Afr = Africa; Am =America; As =Asia; Austr $=$ Australia; Bor $=$ Borealis; Centr $=$ Central; et $=$ And; Geront =Gerontia; Himal=Himalaya; Ind=Indian; Occ $=$ Occidentalis; Or $=$ Oriental; Orient $=$ Oriental; Reg=Region; Subtrop $=$ Subtropical; Temp =Temperate; Trop =Tropical; $*=$ Endemic and $* *=$ Near Endemic

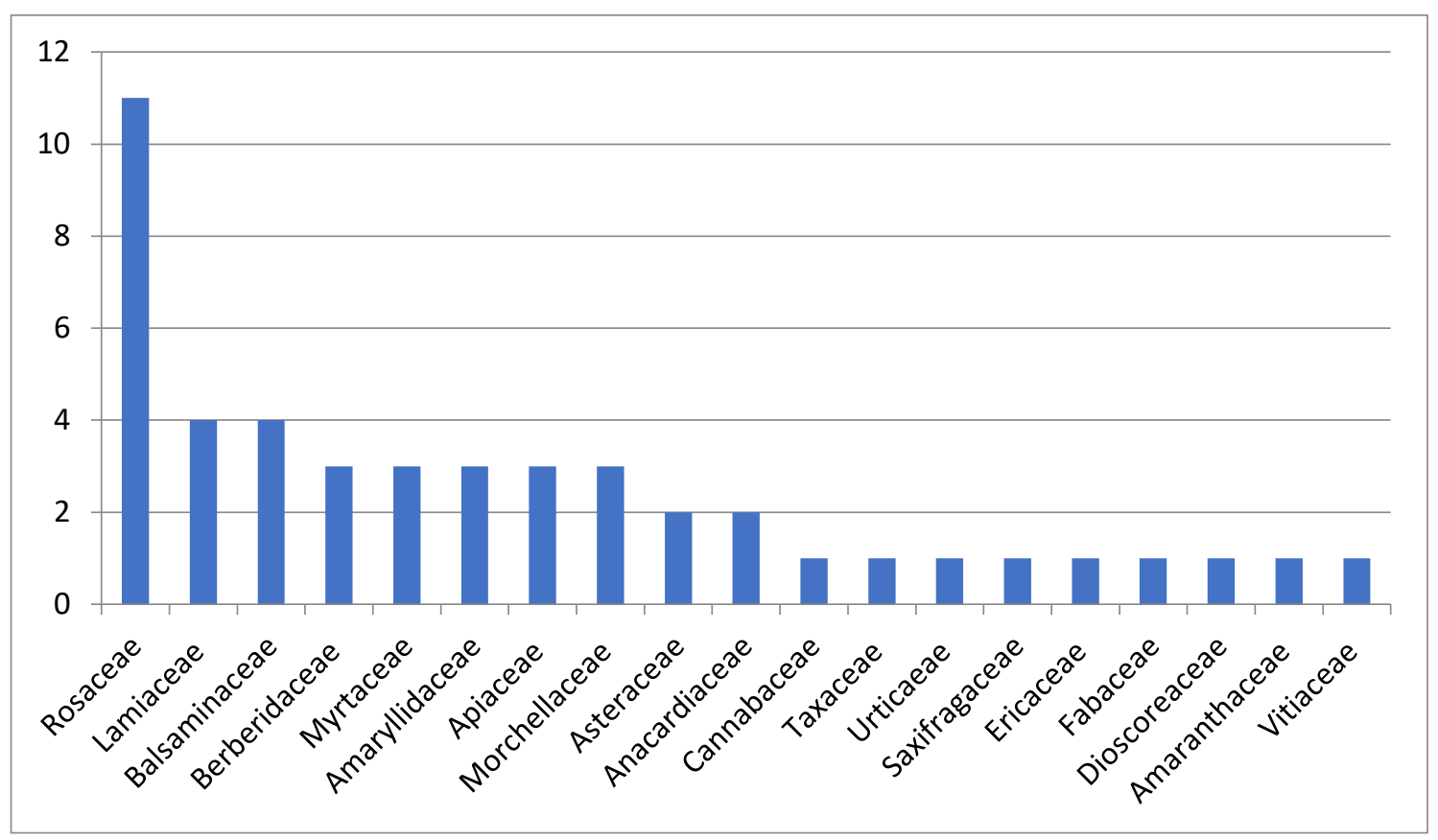

Fig 2: Families recorded.

\section{Results and Discussion}

A total of 58 species of medicinal plants from 27 families and 42 genera were documented. Among them, the most dominant family was Rosaceae and 11others include Amaranthaceae 1, Anacardiaceae 2, Amaryllidaceae 3, Apiaceae 3, Asteraceae 2, Balsaminaceae 4, Berberidaceae 3, Brassicaceae 1, Cannabaceae 1, Dioscoreaceae 1, Elaeagnaceae 1, Ericaceae 1, Fabaceae 1, Grossulariaceae 1, Hippocastanaceae 1, Juglandaceae 1, Lamiaceae 4, Liliaceae 1, Morchellaceae 
3, Myrtaceae 3, Myricaceae 1, Oxalidaceae 1, Polygonaceae 3, Solanaceae 1, Taxaceae 1, Saxifragaceae 1, Vitaceae 1, Urticaceae 1.

Most of the plants such as Morchella sp, Naustratium officinale,Taraxacum officinale, Urtica dioica, Allium sp, Withania somnifera, Curcuma longa, Cannabis, Mentha, Ocimum sanctum are rich in antioxidants and have a wide range of medicinal values used to treat cough, cold, fever, and bronchitis. Ripe fruits of Rubus sp, Berberis Lycium, Elaeagnus parvifolia, Ribes glaciale, syzium cumini, Rhododendron arboretum, Oxalis sp, Morus nigra, Myrica esculenta, Pyrus pashia are loaded with Vitamin A, C, and minerals like Mn, Zn, Mg, etc. All these plants were used traditionally to boost immunity. Due to the covid -19 pandemic, the utilization of these herbal plants has increased many folds in the area. Achyranthes Aspera, Fagopyrum esculentum and Fagopyrum debtors are eaten in processed form (Saag). Juice and chatni are prepared from the flowers of Rhododendron arboretum and Oxalis corniculata.

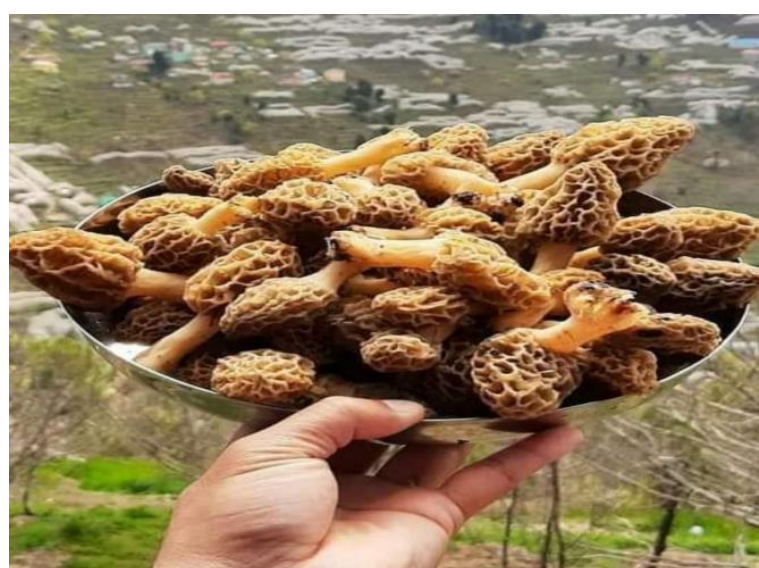

Morchella. esculenta

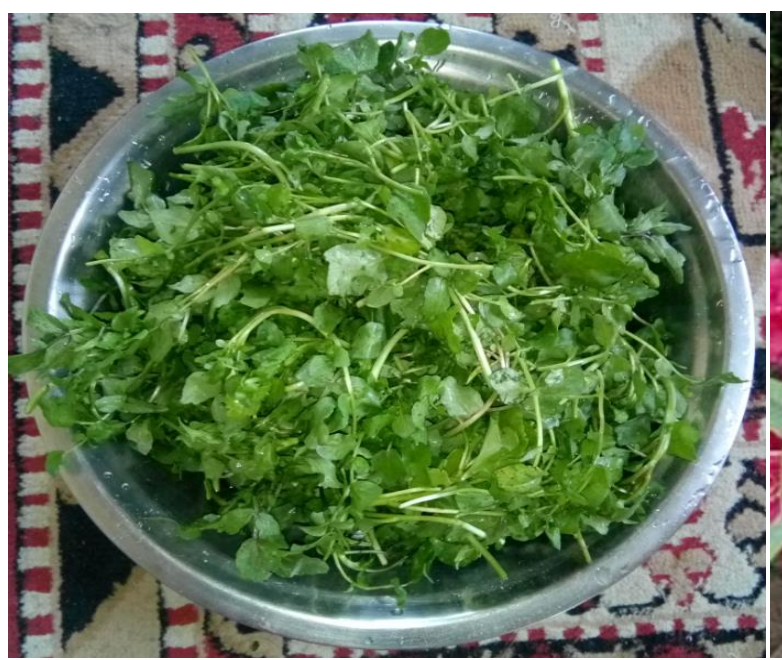

Nasturtium officinale

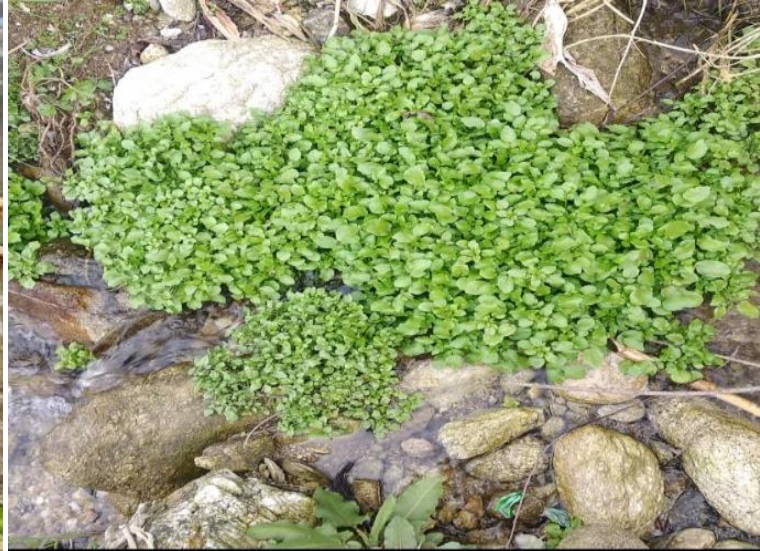

Nasturtium officinale nearby fresh water stream

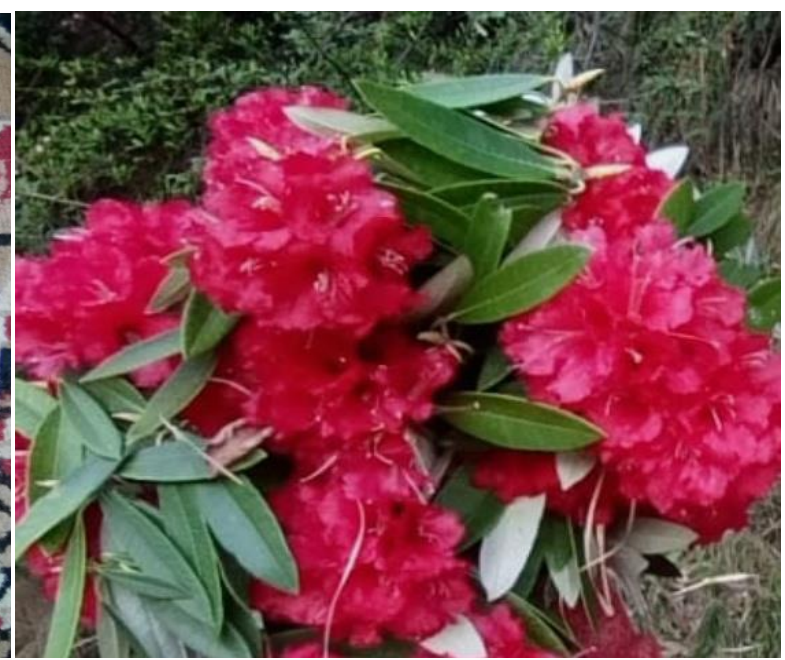

Rhododendron arboretum 


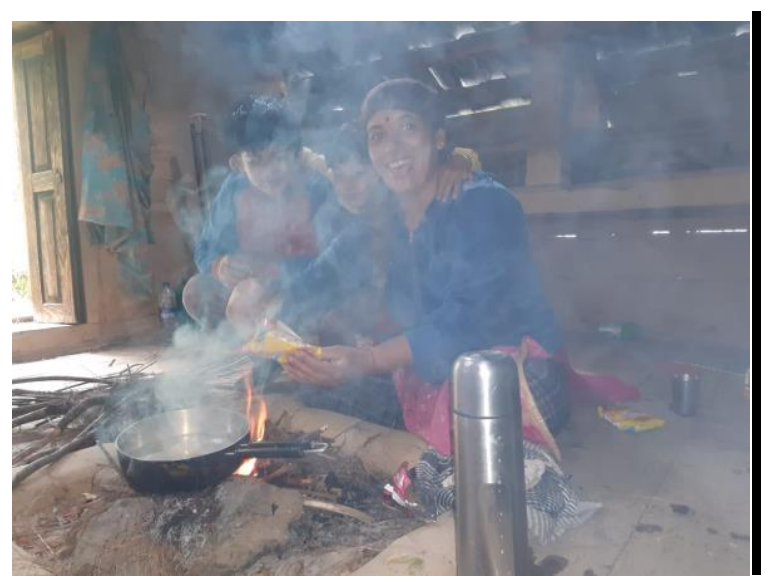

Women using Thymus linearis

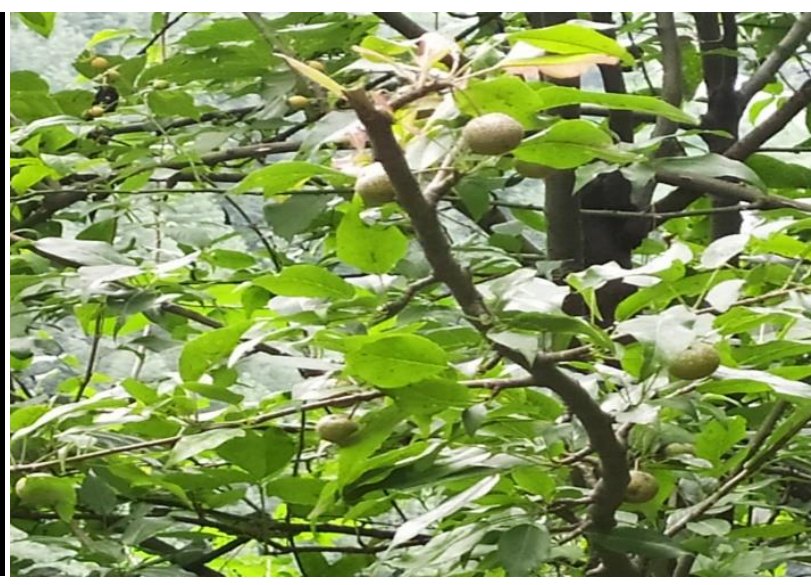

Pyrus pashia
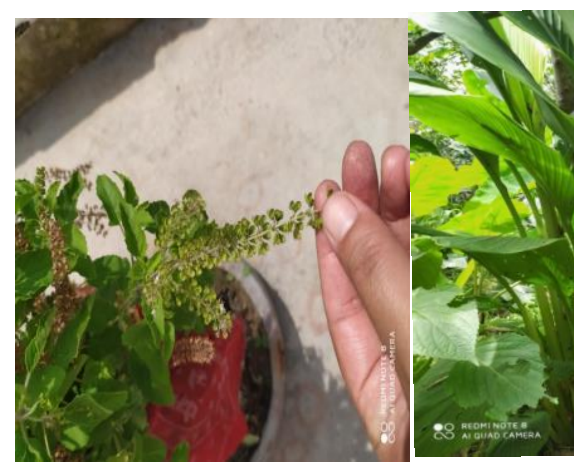

Curcuma longa

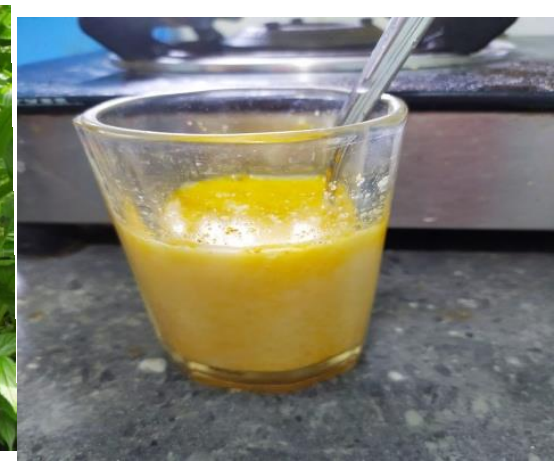

Immunity booster drink (milk with

haldi powder )

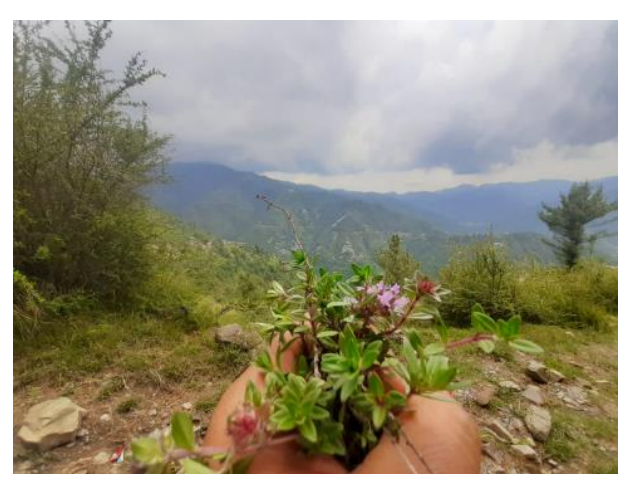

Thymus linearis

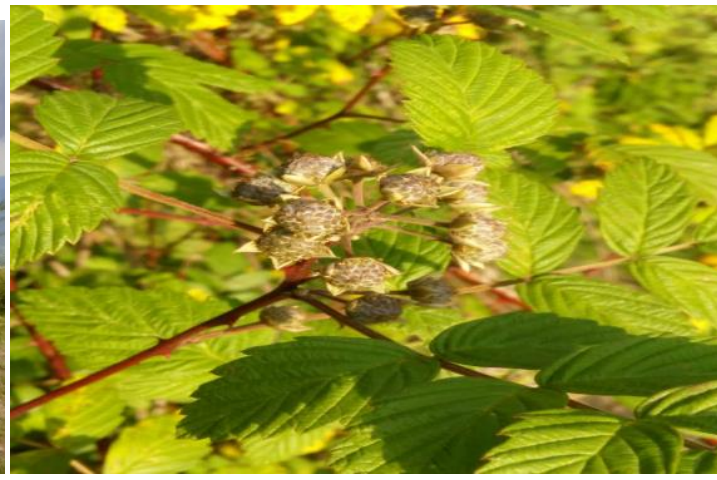

Rubus niveus

52 | 


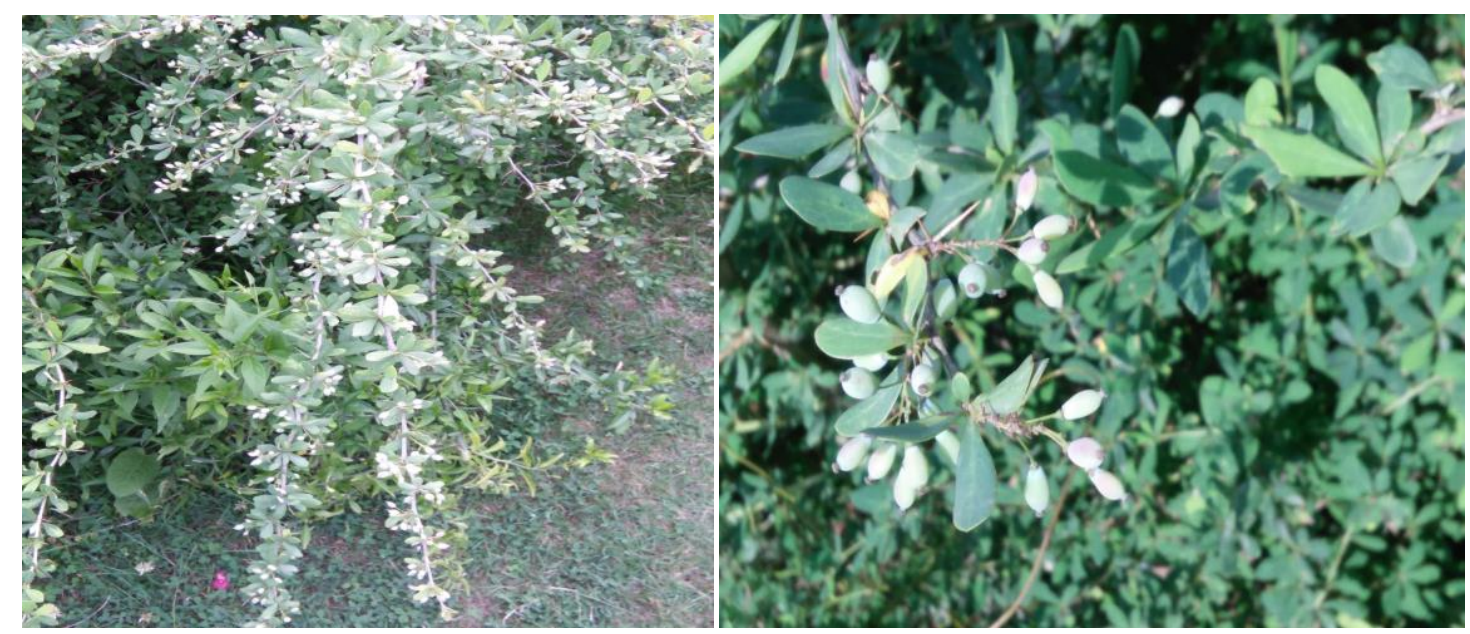

Berberis aristata

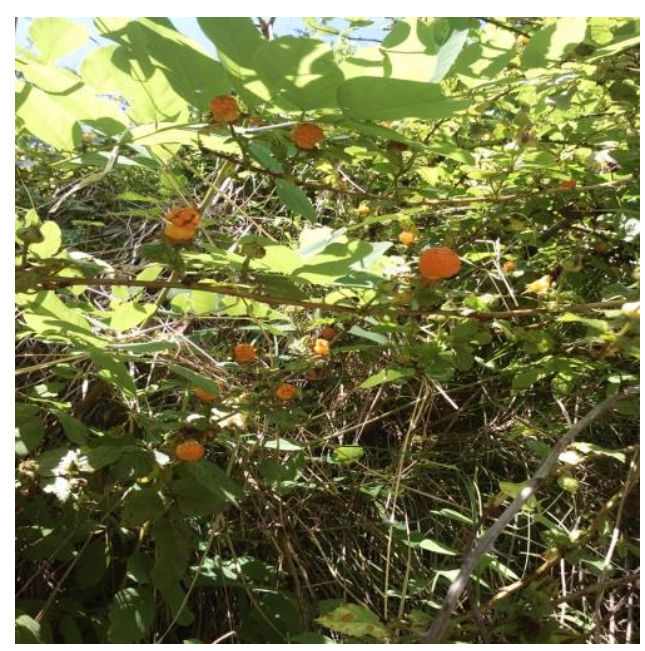

Rubus biflorus

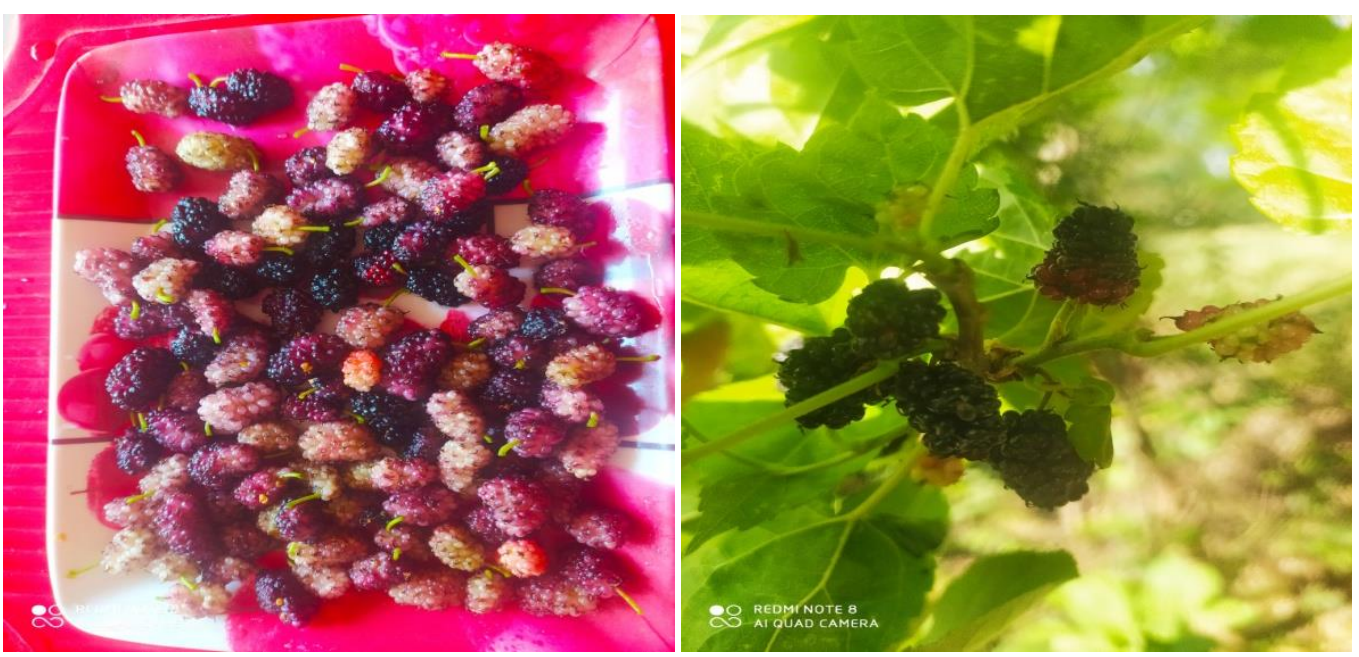

Morus nigra

531 Journal of Scientific Research in Medical and Biological Sciences https://bcsdjournals.com/index.php/jsrmbs 


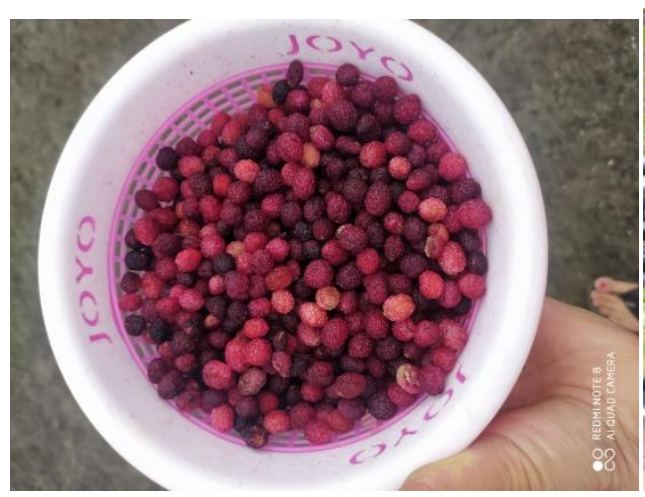

Myrica esculenta

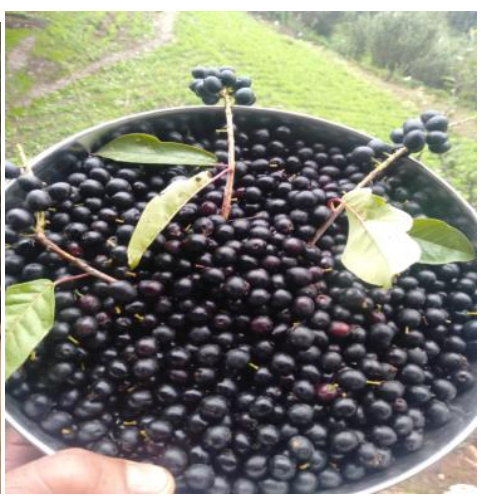

Syzygium cumini fruit

Immunity boosting plants of the study area.

Ever since the safety and clinical characteristics of traditional medicines have come out, their consumption has been gradually elevating after approval by scientific communities (Sarfraz et al., 2020; Rastogi et al., 2020; Rajagopal et al, 2020; Shankar et al., 2020). Attributable to the side-effects of chemical compounds on to human bodies, it has believed to utilize herbal therapies with much more effectiveness and nil side-effects. Understanding the gravity of the situation and health crises, it is the responsibility of the scientific community to look for alternatives or techniques to develop viral vaccination against COVID-19 infection. Having a healthy lifestyle, natural food products can boost the immune functions of the body to combat the severity of viral infections. While improving the immune responses, they also provide resistance against pathogenic organisms (Read., Obeid., Ahlenstiel, Ahlenstiel, 2019; Adhikari et al., 2019; Khadka et al., 2021). Zinc is one of the indispensable elements which has a significant role in the modulation of growth and development and the regulation of the immunomodulatory responses (Kunwar et al., 2019; Prajapati \& Kumar, 2020; Mohammadi\& Shaghaghi, 2020). Deficiency in the $\mathrm{Zn}$ results in enhanced vulnerability to microbial infections specifically viral.

\section{CONCLUSION}

The study found that the use of medicinal plants has increased during COVID-19 and most of the respondents recommended medicinal plants to prevent COVID-19 and to boost immunity. However, there is an urgent need to conduct more studies to come up with appropriate doses and formulations for discovering drugs or vaccines from these formulations. For this purpose, scientific evidence and comprehensive pharmaco-dynamic knowledge related to medicinal plants should be made available to scientists to design clinical trials. Integration of this concept would certainly develop drug therapy in the near future.

\section{ACKNOWLEDGEMENTS}

The authors are thankful to the inhabitants of the area for their kind help and for providing valuable information during the field surveys.

\section{CONFLICTS OF INTEREST}

There are no conflicts of interest 


\section{FUNDING}

No funds were received for completing this study.

\section{REFERENCES}

Adhikari, M., Thapa, R., Kunwar, R. M., Devkota, H. P., \& Poudel, P. (2019). Ethnomedicinal Uses of Plant Resources in the Machhapuchchhre Rural Municipality of Kaski District, Nepal. Medicines, 6(2), 69. MDPI AG. Retrieved from http://dx.doi.org/10.3390/medicines6020069

Arora, R., Chawla, R., Marwah, R., Arora, P., Sharma, R. K., Kaushik, V., Goel, R., Kaur, A., Silambarasan, M., Tripathi, R. P., \& Bhardwaj, J. R. (2011). Potential of Complementary and Alternative Medicine in Preventive Management of Novel H1N1 Flu (Swine Flu) Pandemic: Thwarting Potential Disasters in the Bud. Evidence-based complementary and alternative medicine : eCAM, 2011, 586506. https://doi.org/10.1155/2011/586506

Boulware, D. R., Pullen, M. F., Bangdiwala, A. S., Pastick, K. A., Lofgren, S. M., Okafor, E. C., ... \& Hullsiek, K. H. (2020). A randomized trial of hydroxychloroquine as postexposure prophylaxis for Covid-19. New England Journal of Medicine, 383(6), 517-525. https://doi.org/10.1056/NEJMoa2016638

Keni, R., Alexander, A., Nayak, P. G., Mudgal, J., \& Nandakumar, K. (2020). COVID-19: emergence, spread, possible treatments, and global burden. Frontiers in public health, 8, 216. https://doi.org/10.3389/fpubh.2020.00216

Khadka, D., Dhamala, M. K., Li, F., Aryal, P. C., Magar, P. R., Bhatta, S., Thakur, M. S., Basnet, A., Cui, D., \& Shi, S. (2021). The use of medicinal plants to prevent COVID-19 in Nepal. Journal of ethnobiology and ethnomedicine, 17(1), 26. https://doi.org/10.1186/s13002021-00449-w

Kunwar, R. M., Shrestha, K. P., \& Bussmann, R. W. (2010). Traditional herbal medicine in far-west Nepal: a pharmacological appraisal. Journal of ethnobiology and ethnomedicine, 6, 35. https://doi.org/10.1186/1746-4269-6-35

Lata, M. (2018). Ethnopharmacological studies on the medicinal plants used by semipastoral gujjar tribe in hills of Shikari Devi of Tehsil Thunag, District Mandi Himachal Pradesh. migration, 5, 8.

Lata M and Vijay Kumar(2020). Ethnobotanical study of wild edible plants of janjehli valley mandi district of himachal-pradesh-north-western-himalaya-india. The Journal of Ethnobiology and Traditional Medicine Photon, 131,1525-1537. 2020

Manju Lata(2020). Nutritional, medicinal and indigenous use of Nasturtium officinale in Tehsil Thunag of District Mandi, Himachal Pradesh, North Western Himalayas, India. International Journal of Chemical Studies, 8(5):1648-1653.

https://doi.org/10.22271/chemi.2020.v8.i5w.10535

Mohammadi, N., \& Shaghaghi, N. (2020). Inhibitory effect of eight secondary metabolites from conventional medicinal plants on COVID_19 virus protease by molecular docking analysis. Preprint39. https://doi.org/10.26434/chemrxiv.11987475.v1

Prajapati, S., \& Kumar, N. (2020). SARS-CoV-2 pandemic: an opportunity for Indian traditional medicines (AYUSH). International Journal of Complementary \& Alternative Medicine, 13(3), 103-5.

Raghuvanshi, D., Dhalaria, R., Sharma, A., Kumar, D., Kumar, H., Valis, M., Kuča, K., Verma, R., \& Puri, S. (2021). Ethnomedicinal Plants Traditionally Used for the Treatment of Jaundice (Icterus) in Himachal Pradesh in Western Himalaya-A Review. Plants (Basel, Switzerland), 10(2), 232. https://doi.org/10.3390/plants10020232 
Rastogi, S., Pandey, D. N., \& Singh, R. H. (2020). COVID-19 pandemic: A pragmatic plan for ayurveda intervention. Journal of Ayurveda and integrative medicine, S0975-9476(20)30019X. Advance online publication. https://doi.org/10.1016/j.jaim.2020.04.002

Rajagopal, K., Byran, G., Jupudi, S., \& Vadivelan, R. (2020). Activity of phytochemical constituents of black pepper, ginger, and garlic against coronavirus (COVID-19): An in silico approach. International Journal of Health \& Allied Sciences, 9(5), 43. https://link.gale.com/apps/doc/A626370548/AONE?u=anon 37ba48ef\&sid=googleSchol ar\&xid=7eb960e8

Read, S. A., Obeid, S., Ahlenstiel, C., \& Ahlenstiel, G. (2019). The Role of Zinc in Antiviral Immunity. Advances in nutrition (Bethesda, Md.), 10(4), 696-710. https://doi.org/10.1093/advances/nmz013

Rodgers, W.A., Panwar, H.S.(1988). Planning a wildlife-protected area network in India, 1988.

Sarfraz, I., Rasul, A., Hussain, G., Adem, S., \& Ali, M. (2020). Natural Immune boosters as first-line armours to combat viral infection-COVID19: Myth or Science?. Preprints 2020, 2020030427. https://doi.org/10.20944/preprints202003.0427.v1

Shankar, A., Dubey, A., Saini, D., \& Prasad, C. P. (2020). Role of Complementary and Alternative Medicine in Prevention and Treatment of COVID-19: An Overhyped Hope. Chinese journal of integrative medicine, 26(8), 565-567. https://doi.org/10.1007/s11655-020-2851-y

Singh, K. J., \& Thakur, A. K. (2014). Medicinal plants of the Shimla hills, Himachal Pradesh: a survey. Int J Herb Med, 2(2), 118-127.

Vellingiri, B., Jayaramayya, K., Iyer, M., Narayanasamy, A., Govindasamy, V., Giridharan, B., Ganesan, S., Venugopal, A., Venkatesan, D., Ganesan, H., Rajagopalan, K., Rahman, P., Cho, S. G., Kumar, N. S., \& Subramaniam, M. D. (2020). COVID-19: A promising cure for the global panic. The Science of the total environment, 725, 138277. https://doi.org/10.1016/j.scitotenv.2020.138277 